\title{
On the mechanism of photoinduced phase transitions in ternary liquid crystal systems near thermal equilibrium
}

\author{
Wilson Quevedo, Marcel Petri, Gerhard Busse, and Simone Techert ${ }^{\mathrm{a})}$ \\ Department of Structural Dynamics of (Bio)chemical Systems, Max Planck Institute \\ for Biophysical Chemistry, 37070 Göttingen, Germany
}

(Received 19 December 2007; accepted 19 May 2008; published online 8 July 2008)

\begin{abstract}
According to their phase diagram, polyalkyl glycol ether dissolved in ternary solutions (water, alcane, and cyclohexane) lead to the formation of either liquid crystal phases or microemulsion phases. By photosensitization of the ternary system with laser dyes and choosing the adequate concentration and temperature conditions of these lyotropic systems, it is possible to photoinduce the phase transitions from the microemulsion phase to the liquid crystal phase (and vice versa). The phototransformation conditions were chosen in such a way that the system is in thermal equilibrium during the entire phase transition. The method of photo small angle x-ray scattering has been applied to investigate the mechanism of photoinduced phase transition. Spectroscopically, the mechanism of photoinduced phase transition has been characterized by optical absorption and emission techniques. (C) 2008 American Institute of Physics. [DOI: 10.1063/1.2943200]
\end{abstract}

\section{INTRODUCTION}

In a thermodynamic context, the study of phase transitions is of interest in order to understand the properties of matter as a function of pressure $p$, volume $V$, or temperature $T$, particularly at the $p, V, T$ regime where nonmonotonic dependences are found (first order phase transitions). Phase transitions can coincide with the change in symmetry, the change in magnetic properties, or the change in conductivity, which makes them attractive for material science. The development of lasers has made it possible to introduce phase changes via light irradiation and to control the phase transitions by the absorption of optical photons (as photoinduced phase transitions). In some cases these photoinduced transitions can induce macroscopic changes in the state of the system. ${ }^{1,2}$ With excitation techniques by ultrafast lasers, it is possible to excite solid matter coherently and to drive the solid systems from one state to another by, for example, using light-induced cooperative interactions. ${ }^{3,4}$ So far, the photoinduced phase transitions reported were limited by the systems themselves, and their optical properties mostly occurred as surface effects. The investigated phase transitions were either driven by laser heating or by intermediate formation following optical selection rules. Photosensitization by means of using dye systems extends the possibility of photoinducing phase transitions to any arbitrarily chosen matter, which might be inherently inert against optical excitation. Furthermore, photosensitization allows the observation of phase transitions, which are driven by Stokes or anti-Stokes optical excitation resulting in a heating or cooling of the surrounding environment and as the initiation of a photoinduced phase transition (PIPT).

The system studied in this work is a photosensitized less-ordered microemulsion phase undergoing a PIPT to a highly ordered, self-organized lamellar phase (liquid crystal

${ }^{a)}$ Electronic addresses: stecher@gwdg.de and idreger@gwdg.de. system) or vice versa (Fig. 1, top). According to the phase diagram (Fig. 1, bottom) in ternary microemulsion systems, the phase transition can be thermodynamically introduced by changing the temperature and/or the concentration of the surfactant in the mixture. For the system water, $n$-decane and cyclohexane enriched with varying concentrations of the surfactant polyglycol ether $\mathrm{C}_{8} E_{4}$ (Fig. 1, bottom), schematically summarize the concentration and temperature dependencies. In addition to these thermodynamic parameters, it is possible to photoinduce the phase transition from an otherwise photoinert system like from the optically transparent microemulsion phase to the liquid crystal phase or vice versa by photosensitization and optical excitation, as indicated by the arrows crossing the top line of the tail of the fish diagram.

Due to the amphiphilic character of the surfactant molecules, it is characteristic for nonionic and ionic surfactant systems to be able to solubilize both water and oil molecules.

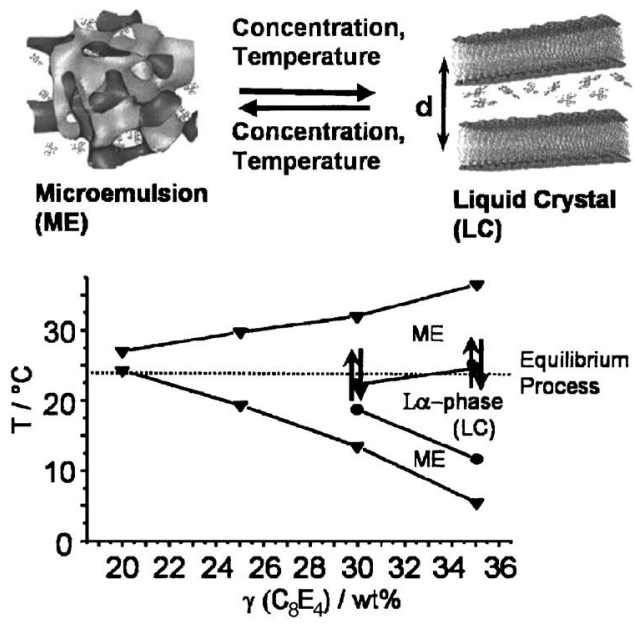

FIG. 1. Schematic drawing of the principle of PIPTs in ternary systems and its phase diagram. Top: structural phase transition; bottom: phase diagram of the system $\mathrm{C}_{8} E_{4}$ in water/octane/cyclohexane. 


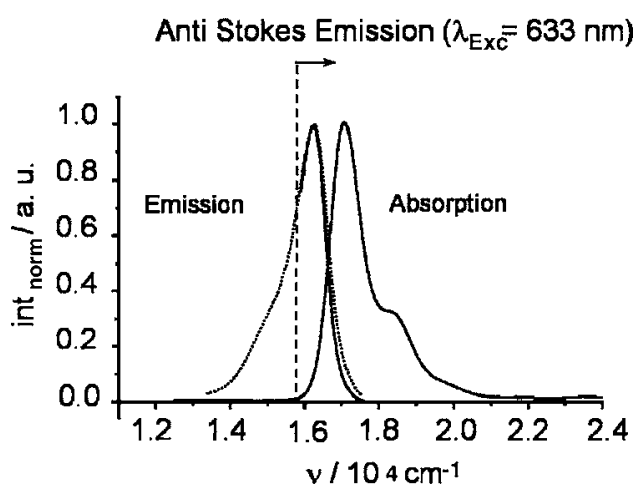

FIG. 2. Absorption and fluorescence emission spectra of Rh101 dyesensitized $\mathrm{C}_{8} E_{4} /$ water/octane/cyclohexane ternary microemulsion system $(\gamma=35 \%)$ demonstrating the principle of optical Stokes and anti-Stokes excitations.

As a consequence, these systems exhibit a variety of thermodynamic stable phases at a macroscopic length with a wide range of domain size from nanometers to micrometers. ${ }^{5,6}$ These phases and their aggregation properties have been studied with special interest in their structure and stability. ${ }^{7,8}$ The self-assembled aggregates exhibit various structures such as spherical micelles, cylindrical micelles, lamellar phases, and bicontinuous structures, just to quote the most common ones (Fig. 1, top left). The microemulsion phase of a ternary mixture is composed of a polar solvent, a nonpolar solvent, and an amphiphilic agent $\left(\mathrm{C}_{n} E_{x}\right)$. At the mesoscopic scale, the simplest approach is to consider the microemulsion phase as a collection of droplets with water and oil domains of colloidal size separated by a layer of surfactant with either micelle or inverse micelle structure. The phase diagram is controlled by the variation of surfactant concentration while keeping the water/oil concentrations constant. The solubility properties of the surfactant systems, and hence their concentration dependence of the phase diagram, are directly related to the bending module of the surfactant agent and can be enhanced by the addition of block copolymers. ${ }^{9-11}$

The microemulsion phase reveals short-range correlation order and is hence a less-ordered phase. On the other hand, in the $L_{\alpha}$ phase also named the liquid crystal phase, molecules self-assemble into aggregates with long-range order correlations (anisotropic fluid), birefringence effects, and other electro-optical and magneto-optical properties. ${ }^{12}$ Liquid crystal phases have been under investigation for almost a century since its discovery by Reinitzer. ${ }^{13,14}$ These phases can be classified by their structure-nematic or smectic (which are the most common phases)_or by the way the phase transition appears, either by concentration changes (lyotropic liquid crystals) or by variation of the temperature (thermotropic liquid crystals). In this study, the liquid crystal phase is a thermotropic smectic assemble with periodic distances in the order of 70-80 А.

To photoinduce the phase transition, the optically inert microemulsion system is sensitized with the dye rhodamine 101 (Rh101) whose fluorescence quantum yield is nearly $1 .^{15,16}$ By tuning the excitation wavelength, the dye can be optically excited in the Stoke or in the anti-Stokes regime, ${ }^{17-19}$ as been indicated in Fig. 2. Figure 2 summarizes the absorption and emission properties of the dye-sensitized microemulsion system for one of the "arrow transitions" in Fig. 1 (bottom) for a surfactant concentration of $\gamma=35 \%$.

The PIPT in the bulk has been studied by means of timeresolved photo small angle X-ray scattering (photo-SAXS), ${ }^{20}$ which was combined with fluorescence spectroscopy. Fluorescence spectroscopy gives information about the local structure around the fluorophore. Photo-SAXS is a new and unique technique to study photoinduced long-range order of the bulk: traditionally $\mathrm{x}$-ray techniques are ideal tools for probing the structure of the bulk material undergoing a phase transition. The combination of both methods (photo-SAXS), lasers for the photoinduction of the phase transition and $\mathrm{X}$-rays for probing the structural reorganization during the phase transition, provides an outstanding and unique tool not only for the detailed study of local phenomena, ${ }^{21-23}$ but also for the study of (cooperative) phenomena in the bulk. ${ }^{1,4}$

SAXS is widely used for the structure determination of nanosized objects as the determination of the radius of gyration of proteins or periodic structures with lattice constants of about 50-100 $\AA$ and bigger. ${ }^{5,6,24,25}$ Since the $d$-spacing in liquid crystal systems studied is in the order of $d_{h}=70 \AA$, the Bragg peak in the small angle regime $\left(q_{\max }=0.06 \AA^{-1}\right)$ is characteristic of the long-range order of the liquid crystal system. Photo-SAXS is one possibility of extending the SAXS technique to the time domain, with the advantage of an initial, optically well-defined, and coherent excitation of the sample. This allows for time-resolved experiments down to time resolution of the apparatus, which might be the read out time of the used detector or, in cases of pulsed optical and pulsed x-ray sources, down to the time resolution of the longest (optical or x-ray) pulse. For time-resolved x-ray diffraction experiments in general, the best time resolution that can currently be achieved is about 100 fs based on an optical laser pump/x-ray probe scheme where the x-ray pulses are either generated in laser plasmas or-at large scale facilities - in a linear collider. ${ }^{26,27}$ In these experiments an optical laser excites the object of investigation, and the photocreated structure is then probed by the detection of the scattered $\mathrm{x}$-ray pulse photons. By varying the time delay between optical laser pump pulse and x-ray probe pulse information about the time evolution of structural changes in the photoexcited matter is obtained. Experiments with the highest spatial resolution, although with lower time resolution of about 30-50 ps, are possible by applying the laser pump/xray probe technique at storage ring facilities like synchrotrons of the third generation. ${ }^{28,29}$ Here, i.e., reorganization processes of molecular crystals can be investigated on the nanosecond time scale. Though the time resolution has been slower than $50 \mathrm{ps}$, the obtained diffraction data were collected with such a high spatial resolution and diffraction peak statistics that they allow for a quite detailed inter- and intramolecular structural analysis of the molecular crystals, making it possible to distinguish between various reaction pathways - at least on the subnanosecond to nanosecond time scale. $^{30}$

For probing the structural dynamics of chromophors in the liquid phase, time-resolved extended $\mathrm{x}$-ray absorption fine structure techniques have been pioneered. ${ }^{31}$ 
Furthermore, on a slower time scale, in the case of home-laboratory-based time-resolved setups, it is also possible to apply the laser pump/x-ray probe technique in a modified experimental approach: in this kind of the setup the laser is synchronized to the read out time of the detector (or vice versa). ${ }^{20}$ Variation of the time delay between optical laser pump and the detector read out time allows for timeresolved experiments down to the (sub) millisecond time scale depending on the time resolution of the detector. Though time resolution and flux are naturally not comparable to external $\mathrm{x}$-ray sources with this apparatus, useful studies on slower transformation processes can be performed.

\section{EXPERIMENTAL SECTION}

\section{A. Sample preparation}

The surfactant agent and polyalkyl glycol ether $\mathrm{C}_{8} E_{4}$ (n-octyltetraoxyethylene. $\mathrm{C}_{16} \mathrm{O}_{4} \mathrm{H}_{34}$ ) was purchased from Fluka (98\% purity) and used without further purification. The surfactant agent was dissolved in a ternary solution at various concentrations. The volume fraction $\varphi$ defined as $\varphi$ $=V_{\text {oil }} /\left(V_{\text {oil }}+V_{\text {water }}\right)$ was maintained at $50 \%$. The range of surfactant concentration at which the phase transition was photoinduced was chosen to be close to room temperature $\left(24-26^{\circ} \mathrm{C}\right)$. The solutions were prepared from bidistillated water (polar solvent), $n$-decane or octane (98\% purity, Fluka) (99\% purity, Fluka), and cyclohexane (99\% purity, Merck). After acidification, the laser dye rhodamine 101 (Refs. 15 and 16) was added to this mixture at concentrations at which excimer formation was avoided (Lambda Physics, without further purification). The last would open another reaction channel leading to the quenching of the fluorescence of the monomer dye. The mixture was stirred at room temperature for $1 \mathrm{~h}$ in a thermostat bath, and then the phases were identified using the polarized screening (PLS) technique. The samples were stored in sealed thin-walled glass capillaries of $1 \mathrm{~mm}$ diameter.

\section{B. Phase diagram}

The PLS technique has been applied to identify microemulsion and liquid crystal phases. The data points of the phase diagram (Fig. 1, bottom) were measured in a thermostat bath (variance $\pm 0.02{ }^{\circ} \mathrm{C}$ ) with $2 \mathrm{ml}$ of sample solutions sealed in quartz tubes. Since the phase transition appeared relatively fast and with a clear separation of the phases, the Lever rule was not used. For the PIPT studies (arrow indicated transitions in Fig. 1, bottom), the composition of the mixtures were chosen in such a way that the phase transition appears close to room temperature with a clear change on the polarized scattering light.

\section{Absorption and fluorescence emission spectroscopy}

Absorption spectra were recorded using a Cary-5E UVvis-NIR spectrophotometer (CVarian Australia Pty Ltd). A detailed description of the experimental conditions can be found elsewhere. ${ }^{14}$ The fluorescence spectra have been mea- sured with a Fluorolog 3-22 (Jobin Yvon-Spex, Munich). For a detailed description of the experimental parameters, see Ref. 32. The spectroscopic characteristics of the PIPT has been investigated by attaching the optical laser systems (He:Ne, Ti:Sa) to the fluorescence spectrometer.

\section{The photo small angle $\mathrm{x}$-ray scattering apparatus}

The systems were studied applying the technique of photo-SAXS, a new technique to study photoinduced longrange order of the bulk from the minutes up to the microsecond time scale (depending on the used detector). ${ }^{20}$ The SAXS experiments have been performed on a Kratky camera setup on which various laser systems have been coupled. For the x-rays $\mathrm{Cu} K \alpha(1.542 \AA)$ radiation was used (detectable $q$-range: $q=0.005-0.1855 \AA^{-1}$ ). For the photoexcitation an optical wavelength of $632.8 \mathrm{~nm}$ was chosen (with about $2.5 \mathrm{~mW}$ laser power). During the measurements the volume between the sample and the detector was kept under vacuum $\left(10^{-2}\right.$ bar $)$ to minimize the scattering from the air. The Kratky camera ${ }^{33}$ was calibrated using silver behenate, which is known to have a well-defined periodic structure $(d$ $=58 \AA$ ). As a sample holder a $1 \mathrm{~mm}$ quartz capillary was used, which was mounted horizontally and was temperature controlled $\left( \pm 0.1{ }^{\circ} \mathrm{C}\right)$. All scattering curves were corrected for the empty capillary signal and the background. More technical details can be found in Ref. 20.

\section{RESULTS AND DISCUSSION}

\section{A. Thermodynamic and optical properties}

The phase diagram of the $\mathrm{C}_{8} E_{4}$ system is shown in Fig. 1. The phase diagram is defined by two variables, the temperature $T$ and $\varphi$, being defined as the volume fraction of the surfactant concentration with respect to the water and oil concentration. Here, two specific volume fractions are defined, first is given by the oil to water ratio as $\varphi=V_{\text {oil }} /\left(V_{\text {oil }}\right.$ $\left.+V_{\text {water }}\right)$. For the investigated systems $\varphi$ is maintained constant at $50 \%$. The second volume fraction $\gamma$ is defined as $\gamma$ $=V_{\text {surf }} /\left(V_{\text {surf }}+V_{\text {oil }}+V_{\text {water }}\right)$. For the presented experiments $\gamma$ is varied between $20 \%$ and $35 \%$ with microemulsion $L_{\alpha}$ liquid crystal phase transition temperatures near room temperature. Since in the head of the determined "fish" diagram three phases coexist, ${ }^{5}$ the composition in the tail of the fish has been used concretely for $\gamma=30 \%$ and $\gamma=35 \%$ in the $\mathrm{C}_{8} E_{4}$ system.

Figure 2 summarizes the optical properties of the Rh101 dye in the microemulsion phase (normalized absorption and emission spectra). The optical excitation conditions were chosen in such a way that the ratio of chromophore to optically exciting photons is about $10000: 1$. The used optical conditions guarantee an optical excitation in the linear regime with slow transformation times. The dye was antiStokes excited at $632.8 \mathrm{~nm}$ (with respect to the dyes fluorescence maximum at about $608 \mathrm{~nm} / 16450 \mathrm{~cm}^{-1}$, Fig. 2). Quantum yield measurements verified that the Rh101 fluorescence quantum yield is still 1.0 in the microemulsion phase, indicating that the dye's local vicinity is polar and most probably the water volume part in the microemulsion phase. 


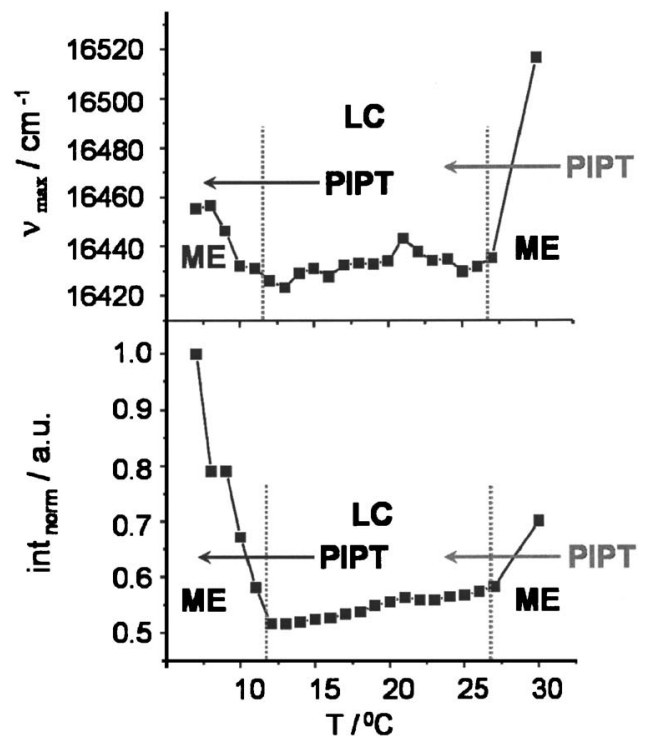

FIG. 3. Fluorescence temperature emission spectra for $\mathrm{C}_{8} E_{4}(\gamma=35 \%)$. Top: shift of the Rh101 emission maximum as a function of temperature. Bottom: temperature dependence of the Rh101 integral fluorescence intensity. For low temperatures $\left(T=10^{\circ} \mathrm{C}\right)$, in the microemulsion phase, the quantum yield of the Rh101 dye is near unity. The left side arrow indicates the PIPT, which has been studied in the following fluorescence experiments. The right side arrow indicates the PIPT, which has been studied in the following time-resolved $\mathrm{x}$-ray small angle diffraction experiments.

\section{B. Photoinduced phase transition: Spectroscopic evidence}

The fluorescence behavior of the Rh101 dye is quite complex by going from the microemulsion (ME) phase over the liquid crystal (LC) phase to the ME phase by temperature increase. In the microemulsion phase, far away from the transition temperature, the quantum yield of the Rh101 dye is nearly 1 . The dye was again anti-Stokes excited at $632.8 \mathrm{~nm}$. As excitation wavelength for the detection of the fluorescence $\lambda_{\mathrm{exc}}=585 \mathrm{~nm}$ has been chosen, which is near to the vibronic (0-0) transition of the electronic $S_{1} \leftarrow S_{0}$ excitation $(600 \mathrm{~nm})$. In order to photoinduce the phase transition, the fluorophore is anti-Stokes excited causing an energy redistribution process in the surrounding environment ${ }^{17,18}$ and guaranteeing a homogeneous optical excitation through the whole capillary system. Figure 3 summarizes the changes of the fluorescence properties of the dye-enriched $\mathrm{C}_{8} E_{4}$ system-concerning the bathochromic and hypsochromic shift of the fluorescence band maximum $\nu_{\max }$ as a function of temperature. In the temperature range, where the liquid crystal phase is formed, $\nu_{\max }$ is shifted to lower energies by about $\Delta \nu_{\max }=40 \mathrm{~cm}^{-1}$. Also, the fluorescence intensity does decrease and increase by going through the ME-LC-ME temperature range. In the ME phase at about $6{ }^{\circ} \mathrm{C}$, the fluorescence quantum yield is about 1 . As indicated by the arrow and the PIPT assignment, at $12{ }^{\circ} \mathrm{C}$ the $\mathrm{ME} \rightarrow \mathrm{LC}$ phase transition occurs and the Rh101 fluorescence quantum yield decreases by a factor of 2 . The intensity decrease is explained by an increase in the structural order in the liquid crystal phase (compared to the microemulsion) leading to an effective volume contraction: Hence, when going from the microemulsion phase to the liquid crystal phase, the structure of

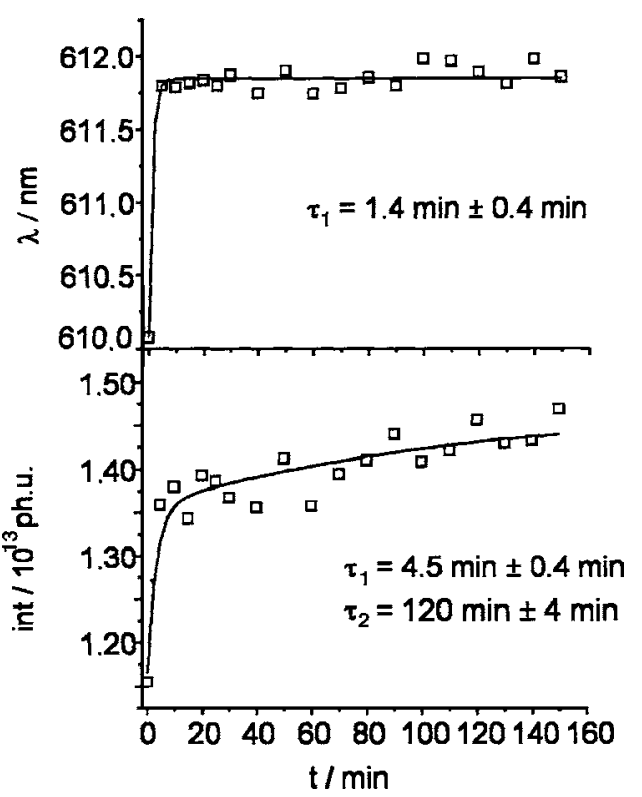

FIG. 4. Time evolution of the emission maximum (top) and the integrated fluorescence intensity (bottom) of Rh101 dye-sensitized $\mathrm{C}_{8} E_{4}$ /water/octane/cyclohexane ternary system $(\gamma=35 \%)$. At the used optical photon fluxes, the phase transition takes place after 4 min of illumination as can be seen as a nonmonotonic rise of the integrated fluorescence intensity by crossing the phase diagram from the liquid crystal phase to the microemulsion.

the ternary system reorganizes. In the microemulsion phase the oil molecules are loosely connected with water surrounding the microemulsion cavities. At the water/oil interface the microemulsion cavities have an average diameter of $180 \AA$. In the surrounding water the Rh101 dye is dissolved (Fig. 1, top left) as indicated by the bathochromic shift of the fluorescence maximum of Rh101. In the smectic liquid crystal $L_{\alpha}$ phase, oil molecules and water interlayers alternatively stack in a more ordered way (Fig. 1, top right) and the effective volume of the surrounding of the dye decreases. Due to this effective "volume contraction" within the water volume, the average distance between the dye molecules decreases in the liquid crystal phase (compared to the microemulsion phase), and with a higher probability Rh101 excimers are formed. The formation leads to a 50\% quenching of the dye fluorescence intensity.

For the time-dependent spectroscopic studies the PIPT from 12 to $11.5^{\circ} \mathrm{C}$ has been studied, as indicated by the transition arrow in Fig. 3. In Fig. 4 the time-dependent fluorescence characteristics of this PIPT from the $L_{\alpha}$ phase to the microemulsion phase are shown. The microemulsion phase was stabilized at $T=12{ }^{\circ} \mathrm{C}$, about $\Delta T=0.5^{\circ} \mathrm{C}$ away from the phase transition temperature (the temperature stabilization was set to be $\Delta T \pm 0.02{ }^{\circ} \mathrm{C}$ ). The optical excitation of the dye-enriched microemulsion phase at $632.8 \mathrm{~nm}$ leads to a comparable intensity increase, as has been observed in the temperature scan of Fig. 3. Note that optical excitation results in a small effective cooling of the system; therefore the phase transition is driven from the higher temperature $L_{\alpha}$ liquid crystal phase to a microemulsion phase. Under the applied excitation conditions, the PIPT occurs within the first 20 min of illumination. It can be spectroscopically monitored by a redshift of the Rh101 fluorescence maximum from 
610 to $612 \mathrm{~nm}$. The shift can be fitted monoexponentially (applying the Levenberg-Marquardt algorithm) with a decay constant of $\tau=1.4 \pm 0.6 \mathrm{~min}$. The integral intensity increase follows a biexponential behavior with growing constants of $\tau_{1}=4.5 \pm 0.4 \mathrm{~min}$ and $\tau_{2}=120 \pm 4 \mathrm{~min}$ (the amplitudes are $-A_{1}=0.11 \pm 0.03$ and $\left.-A_{2}=0.19 \pm 0.03\right)$. The frequency shift of the fluorescence maximum does not show this biexponential behavior, which will be explained later.

\section{Photoinduced phase transition: Evidence in structural and kinetic behavior of $x$-ray scattering}

In Fig. 3, the right side arrow indicates the PIPT studied by time-resolved small angle x-ray diffraction techniques. The observed PIPT occurs from the microemulsion phase stabilized at higher temperatures to the liquid crystal phase at about $26^{\circ} \mathrm{C}$ (near room temperature). Here, the photoinduction leads to an increase in the liquid crystal domains with long-range order, which have been investigated as a reflection in the small angle $\mathrm{x}$-ray regime. This peak is also sensitive to broadening effects due to possible thermal excess energy given in the system by the PIPT mechanism.

Figure 5 (top) presents a three-dimensional plot of the observed PIPT in a ternary system with a surfactant concentration of $\gamma=35 \%$. The scattering intensity maximum of the microemulsion diffuse scatter was found to be at $q_{\max }$ $=0.04 \AA^{-1}$. After $100 \mathrm{~min}$ illumination the diffuse scatter signal is nearly diminished and a new liquid crystal reflection at $q_{\text {max }}=0.085 \AA^{-1}$ has been formed. Figure 5 (bottom) presents the time-dependent behavior of the ternary system without dye sensitization. Essentially no major intensity changes on the microemulsion peak or the occurrence of a sharp reflection indicating the formation of a liquid crystal phase has been observed under optical illumination. The comparison clearly reveals that the PIPT only occurs if the ME system is dye sensitized.

Figure 6 (top) shows a typical SAXS scattering signal before illumination. Figure 6 (bottom) shows the scattering signal of the phototransformed material after $80 \mathrm{~min}$ of illumination time as directly measured with the before described TR photo-SAXS setup. Note that the phototransformation does not lead to a $100 \%$ of phase transformation due to photodegradation effects occurring after $300 \mathrm{~min}$ of laser exposure. As can be clearly seen after 80 min only small residues of the microemulsion phase are left. Compared to the initial scattering intensity of the microemulsion diffuse scatter before illumination, the ME scattering intensity is reduced by nearly $90 \%$. The intensity scattering maximum of the photocreated liquid crystal phase has been found to be at $q_{\max }$ $=0.085 \AA^{-1}$. The ratio of the integral microemulsion scattering intensity to the integral liquid crystal scattering intensity is about $0.1: 1$, indicating that the PIPT to a pure liquid crystal phase was nearly completed under the used excitation conditions.

In the following, a detailed analysis of the time dependence of the SAXS scattering pattern and the structural changes underlying the PIPT will be given. Before light illumination, the ternary system was stabilized in the pure microemulsion phase (Fig. 6, top) whose initial parameter set-
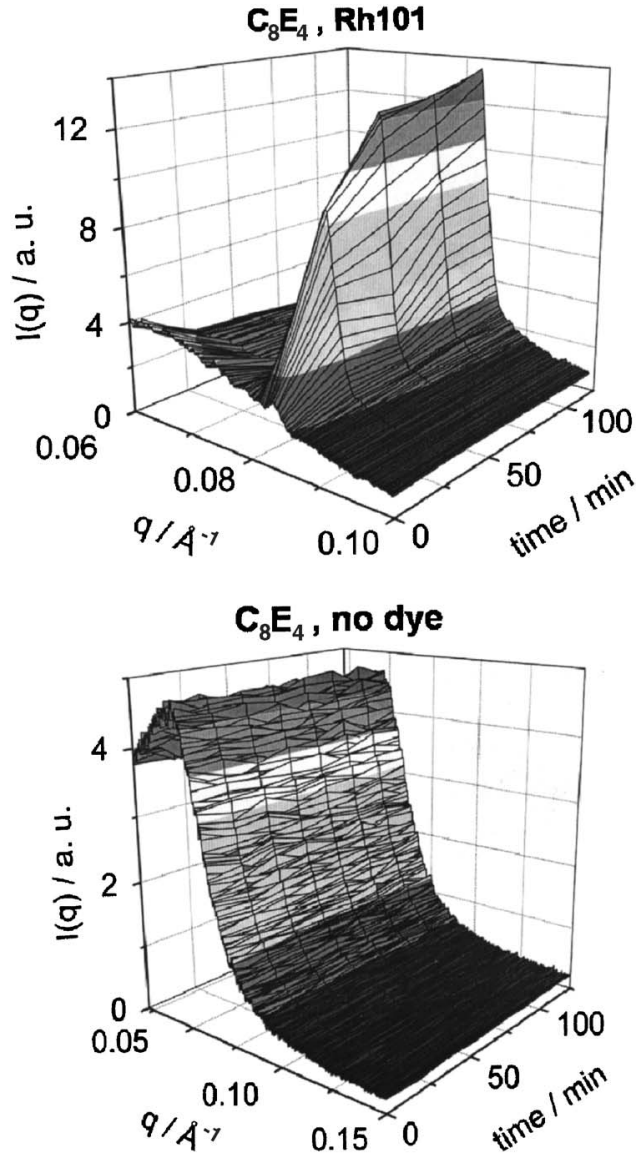

FIG. 5. PIPT from a Rh101 dye-sensitized microemulsion to a liquid crystal system in the system $\mathrm{C}_{8} E_{4} /$ water/octane/cyclohexane $(\gamma=30 \%)$ investigated by time-resolved SAXS. The structural phase transition occurs within the first 10-15 min of illumination coinciding with the spectroscopic findings. The excitation conditions were chosen in such a way that the system thermally had time to equilibrate. Top: PIPT of the dye-sensitized system. Bottom: no PIPT can be observed if the system is not dye sensitized (reference measurement).

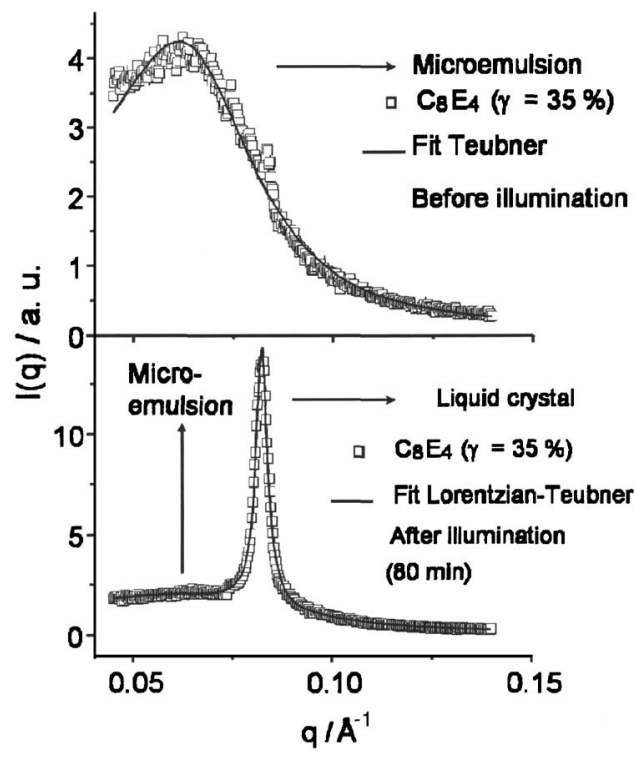

FIG. 6. Top: SAXS pattern from the pure microemulsion phase before illumination. Bottom: SAXS pattern of the nearly pure liquid crystal phase after $80 \mathrm{~min}$ of illumination (system: $\mathrm{C}_{8} E_{4} /$ water/octane/cyclohexane, $\gamma=35 \%$ ). 
TABLE I. Integral x-ray scattering intensities (int), thickness of the lamella $(L)$, number of lamella [No. (lamella)], and lattice constants $\left(d_{\mathrm{LC}}\right)$ of the LC phase as a function of flux. Here number of optical photons absorbed [No. (photons)].

\begin{tabular}{lcccc}
\hline \hline No. (photons) $/ 10^{18}$ & $\begin{array}{c}\text { Int/ } 10^{-5} \\
\text { (e.u. }\end{array}$ & $L(\AA)$ & No. (lamella) & $d_{\mathrm{LC}}(\AA)$ \\
\hline 0 & 0 & 0 & 0 & 0 \\
9.241 & $1.00 \pm 0.18$ & $48.0 \pm 0.76$ & $17 \pm 2$ & $76.90 \pm 0.06$ \\
18.482 & $1.84 \pm 0.26$ & $48.1 \pm 0.47$ & $17.99 \pm 2$ & $77.31 \pm 0.47$ \\
27.723 & $9.86 \pm 0.21$ & $47.9 \pm 1.06$ & $18 \pm 2$ & $76.75 \pm 1.06$ \\
36.964 & $1.11 \pm 0.25$ & $48.0 \pm 1.14$ & $18 \pm 2$ & $76.77 \pm 1.14$ \\
\hline \hline
\end{tabular}

tings can be found in Table I (first line, before illumination). Figure 6 (bottom) shows the formed liquid crystal/ microemulsion phase after $80 \mathrm{~min}$ of light illumination. The observed signal changes, which reflect the composition changes of the mixtures from the microemulsion phase to liquid crystal phase, can be quantitatively treated as a function of time. In a first approach, the liquid crystal diffraction peak (Fig. 6, top) can be described by a Lorentzian function. The Lorentzian function is convoluted with the well known Teubner-Strey equation (Fig. 6, bottom), which describes the behavior of the microemulsion diffuse scatter signal. ${ }^{34}$

Within the kinematic approach, for every time point $t$ the microemulsion diffuse $\mathrm{x}$-ray scattering signal is defined as

$$
I(t) \propto \frac{1}{a_{2}+c_{1}(t) q^{2}+c_{2}(t) q^{4}}+I_{\mathrm{BKG}},
$$

where $I_{\mathrm{BKG}}=$ background scatter signal, $a_{2}$ is a timeindependent scaling factor, and $c_{1}(t)$ and $c_{2}(t)$ are the concentrations of the two components, oil and water (see Sec. II A). The photoinduction of the phase transition is thermotropic (vertical excitation in the phase diagram in Fig. 1) resulting in a redistribution of the concentrations of the components oil and water within the bulk to the liquid crystal phase. Therefore, $c_{1}=c_{1}(t)$ and $c_{2}=c_{2}(t)$. From Eq. (1) the correlation length $\xi(t)$ and the dispersion $d(t)$ with the quasiperiodic repeat distance $d(t) / 2 \pi$ can be extracted to

$$
\xi(t)=\frac{1}{\sqrt{\frac{1}{2} \sqrt{\left(\frac{a_{2}}{c_{2}(t)}\right)}+\left(\frac{c_{1}(t)}{4 c_{2}(t)}\right)}}
$$

and

$$
\frac{d(t)}{2 \pi}=\frac{1}{\sqrt{\frac{1}{2} \sqrt{\left(\frac{a_{2}}{c_{2}(t)}\right)}-\left(\frac{c_{1}(t)}{4 c_{2}(t)}\right)}} .
$$

According to Table I, for the microemulsion phase in Fig. 6 (bottom), it has been found that the periodic repeat unit of the microemulsion is $d=165.21 \pm 7.4 \AA \quad\left(q_{\max }\right.$ $=0.0408 \pm 0.0021 \AA^{-1}$ ) and that its scattering peak halfwidth is $\Delta q_{\mathrm{FWHM}}=0.0055 \pm 0.0002 \AA^{-1}$.

Figure 7 visualizes the coexistence of the microemulsion and liquid crystal phase after $20 \mathrm{~min}$ of laser exposure. The experimental scattering data were fitted by applying the presented convolution of a Lorentzian function with the

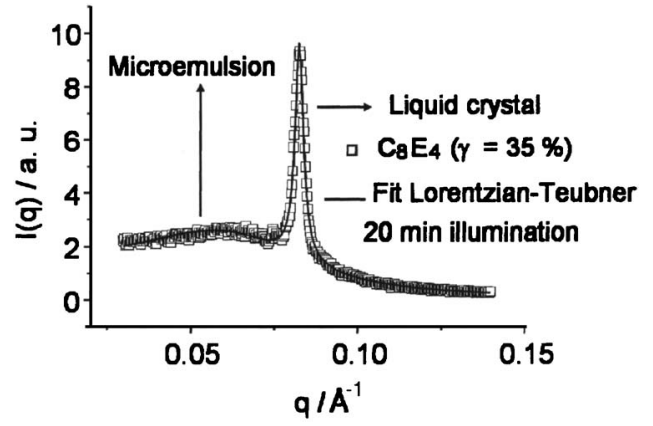

FIG. 7. Coexistence of microemulsion and liquid crystal phase at $t=20 \mathrm{~min}$ (system: $\mathrm{C}_{8} E_{4} /$ water/octane/cyclohexane, $\gamma=35 \%$ ). The experimental scattering data were fitted by applying a convolution of a Lorentzian function (describing the liquid crystal diffraction peak) with the well-known Teubner-Strey equation (describing the microemulsion diffuse scatter signal). After $300 \mathrm{~min}$ of light illumination, the transformation from a pure microemulsion phase to a coexistent liquid crystal/microemulsion phase has taken place.

Teubner-Strey equation emphasizing the validity of the fitting model even for coexistent phases at appropriate times.

Figure 8 shows the results of the final fitting of the liquid crystal reflection with a surfactant concentration of $\gamma=35 \%$, after the subtraction of the background and the microemulsion diffuse scatter signal and after 120 min of photoillumination. The data were quantitatively analyzed within the framework of a Bessel function of first order (Porod equation ${ }^{33}$ ). There time dependence is defined as

$$
q^{4} I(q, t) / I^{0} \approx \sin ^{2}[L(t) q / 2] \frac{\sin ^{2}[N(t) d(t) q / 2]}{\sin ^{2}[d(t) q / 2]},
$$

where the parameters are defined as $I^{0}=8 \pi N_{\text {cryst }} A\left(\rho_{m}-\rho_{p}\right) d$, $N=$ number of lamellae, $d=$ spacing, $L=$ thickness, $A$ $=$ surface of the lamellae, $N_{\text {cryst }}=$ number of (identical) liquid crystals, $\rho_{m}=$ electron density of the interlayer, and $\rho_{p}$ $=$ electron density of the lamella layer. In the following it is assumed that the electron density changes as a function of time cannot be resolved by the resolution of the scattering momentum provided by the home laboratory Kratky apparatus. Therefore these parameters (as well as all the other product parameters of $I^{0}$ ) were kept constant. As time-dependent parameters were refined, $L=L(t), N=N(t)$, and $d=d(t)$.

The results of this refinement for the liquid crystal reflection after 120 min light illumination are summarized in

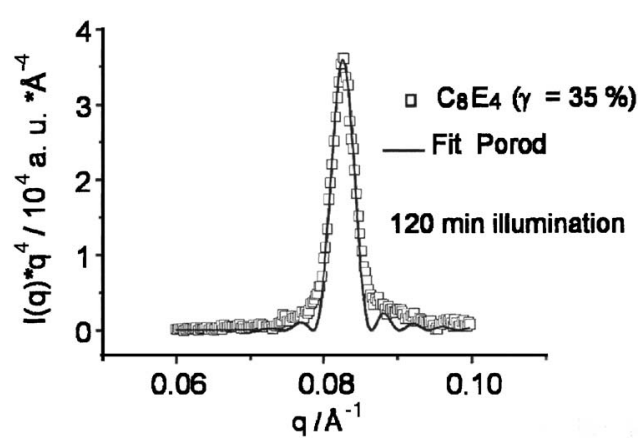

FIG. 8. Porod fitting of the liquid crystal reflection allows for the determination of several experimental structural parameters as a function of time, such as the lamellar periodic unit $d$, the thickness of the lamella $L$, and the number of lamella $N$ [Eq. (4)]. 

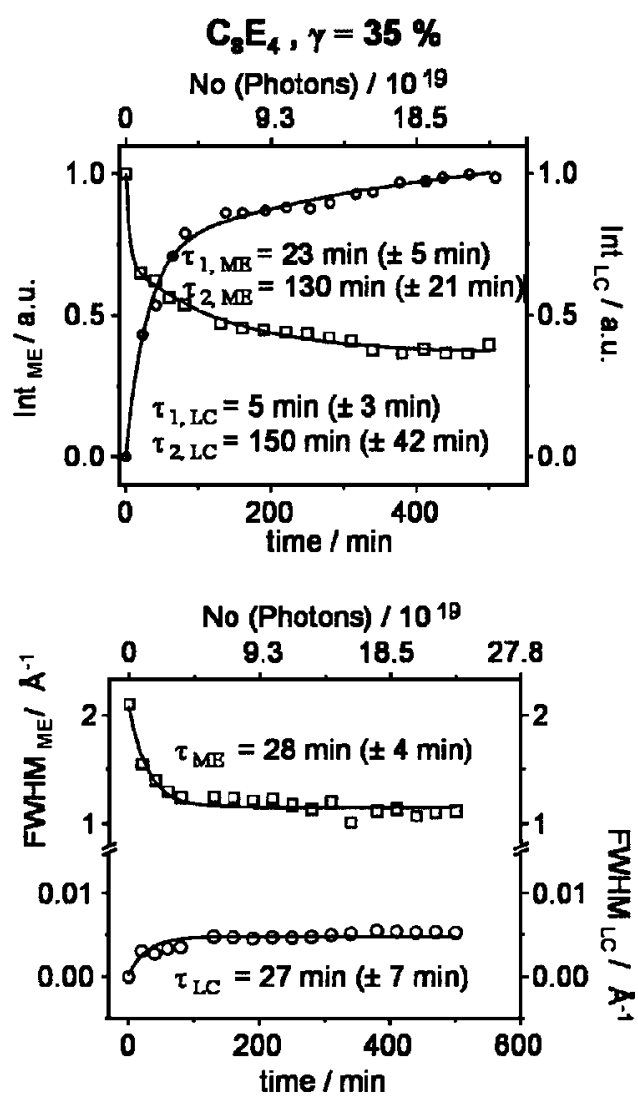

FIG. 9. System: $\mathrm{C}_{8} E_{4} /$ water/octane/cyclohexane, $\gamma=35 \%$. Top: As a function of irradiation time, the normalized integral scattering intensity of the liquid crystal reflection exponentially increases while the normalized integral scattering intensity of the microemulsion diffuse scatter signal decreases. Bottom: time dependence of the full width at half maximum of the ME diffuse scatter signal and the liquid crystal reflection.

Table I (bottom line). After phototransformation the number of formed liquid crystal lamella domains is increased to $N=12$. The domain width is constrained to $L=48.0 \pm 2.2 \AA$ at a maximum of scattering reflection at $q_{\max }$ $=0.085 \pm 0.0003 \AA^{-1}$ and a periodic repeat unit of $d$ $=74.0 \pm 0.3 \AA$.

The time evolution of the whole PIPT process and the underlying structural parameter changes are summarized in Table I in general. Figure 9 (top) visualizes these results as time evolution of the found photoinduced integral scattering intensity changes of the $\mathrm{C}_{8} E_{4}$ ternary system $(\gamma=35 \%)$. For normalization purposes the intensity of the microemulsion phase before light illumination $(t=0 \mathrm{~min}$, left-hand side of Fig. 9) and the intensity of the liquid crystal phase after long illumination time ( $t=500 \mathrm{~min}$, right-hand side of Fig. 9, top) have been normalized to 1 . The integral intensity changes during the PIPT follow a nonmonotonic dependency. The intensity decrease of the microemulsion phase follows a biexponential time law with $\tau_{1, \mathrm{ME}}=23 \pm 5 \mathrm{~min}, \tau_{2, \mathrm{ME}}$ $=130 \pm 21 \mathrm{~min}$ and the amplitudes of $A_{1}=0.41 \pm 0.06, A_{2}$ $=0.57 \pm 0.06$. The growing in of the LC reflection follows a biexponential growing function with $\tau_{1, \mathrm{LC}}=5 \pm 3 \mathrm{~min}, \tau_{2, \mathrm{LC}}$ $=150 \pm 42 \mathrm{~min}$ and $A_{1}=0.32 \pm 0.02, A_{2}=0.32 \pm 0.01$. Also here, Levenberg-Marquard fit algorithms were applied. Most of the LC phase is therefore formed at very fast times after 20-25 min. This corresponds to a maximum photon entry of

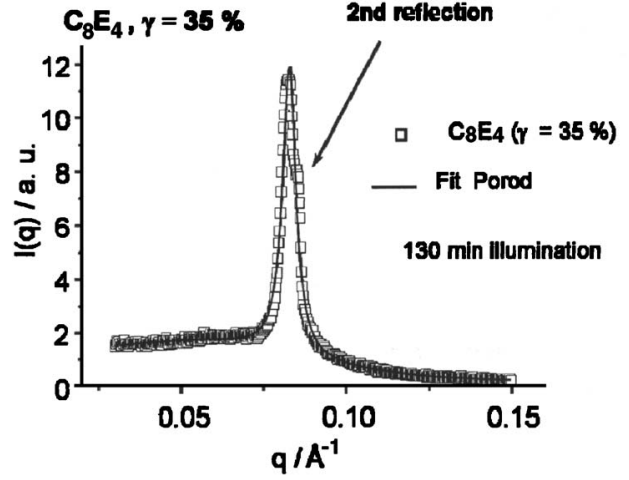

FIG. 10. System: $\mathrm{C}_{8} E_{4} /$ water/octane/cyclohexane, $\gamma=30 \%$. Top: As a function of irradiation time, the normalized integral scattering intensity of the liquid crystal reflection exponentially increases while the normalized integral scattering intensity of the microemulsion diffuse scatter signal decreases. Bottom: time dependence of the full width at half maximum of the ME diffuse scatter signal and the liquid crystal reflection.

about no( $\mathrm{ph})=4.5 \times 10^{19}$ and compared to the dye concentration of a maximum photon excess of no(ph):no(dye) $=10^{4}: 1$ in the considered illumination volume. Taking into account the absorption coefficient at the excitation wavelength, this ratio decreases by a factor of approximately 100 . Quite early, at about $t=50 \mathrm{~min}$, the quasistationary conditions between the two phases are reached corresponding to a photon entry of no(ph) $=2 \times 10^{19}$.

The slow time constants $\tau_{2, \mathrm{ME}}$ and $\tau_{2, \mathrm{LC}}$ can clearly be assigned to a phase separation process within the photoformed LC $L_{\alpha}$ phase to two LC phases with similar periodicity. Figure 10 (after 130 min of illumination, same system) demonstrates this phase segregation behavior by the duplication of Bragg reflection to two peaks (corresponding to two LC phases), which, however, cannot be resolved according to the Nyquist theorem. Nonetheless, these two peaks, which strongly overlap, are also responsible for the quite broad linewidth of the liquid crystal reflection. One should point out that this behavior is quite unusual and shows the complexity of the chemical system, which is created after the PIPT.

The full width at half maximum of the diffuse scatter signal can best be fitted by a monoexponential time law with $\tau_{\text {FWHM,ME }}=28 \pm 4$ min (Fig. 9, bottom). Hereby, the quite broad FWHM of the diffuse scatter reduces from $\Delta q_{\mathrm{FWHM}}$ $=2 \AA^{-1}$ to $\Delta q_{\mathrm{FWHM}}=1 \AA^{-1}$. With a similar time constant $\left(\tau_{\mathrm{FWHM}, \mathrm{LC}}=27 \pm 7 \mathrm{~min}\right)$ the FWHM of the LC reflection increases to $\Delta q_{\text {FWHM }}=0.01 \AA^{-1}$. Its unusual high value indicating a broad diffraction peaks is due to PIPT-induced growing of at least two liquid crystal phases out of the microemulsion phase.

Figure 11 summarizes the time evolution of the found photoinduced integral scattering intensity changes of the $\gamma$ $=30 \% \mathrm{C}_{8} E_{4}$ system. Again, ME integral intensity and LC integral intensity are normalized to 1 for early respective late time points (Fig. 11, top). The decrease in the ME phase intensity can be best fitted monoexponentially with a time decay constant of $\tau_{\mathrm{ME}}=18 \pm 4 \mathrm{~min}$. A biexponential fit leads to an overdescription of the time law. The LC intensity increase follows a biexponential growing time law with $\tau_{1, \mathrm{LC}}$ 


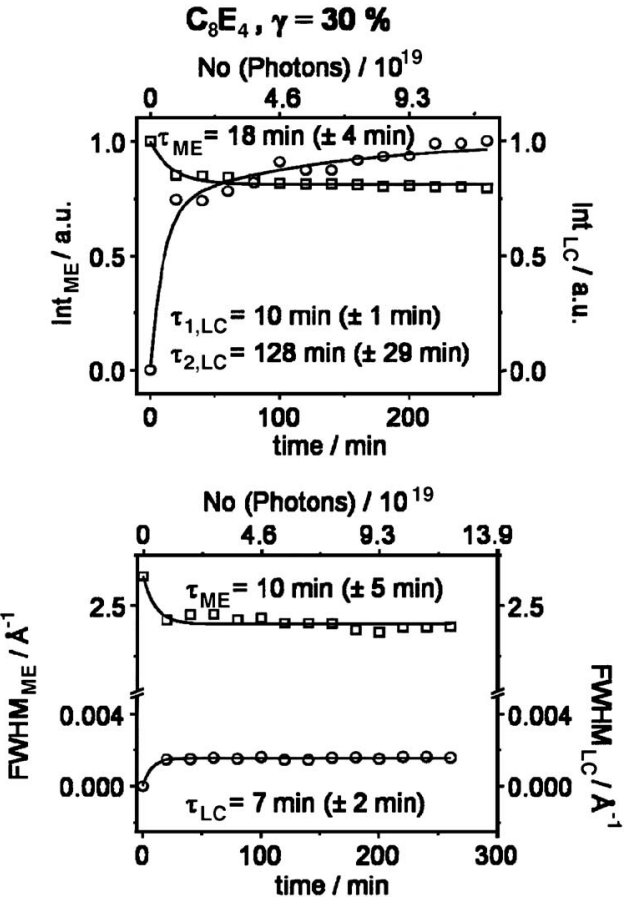

FIG. 11. System: $\mathrm{C}_{8} E_{4} /$ water/octane/cyclohexane, $\gamma=35 \%$. Top: During the formation of the liquid crystal phase, the number of lamella increases from 0 to 16. Bottom: variation of $d$-spacing of the LC phase as a function of illumination time.

$=128 \pm 29 \mathrm{~min}, \tau_{2, \mathrm{LC}}=10 \pm 1 \mathrm{~min}$ and $A_{1}=0.28 \pm 0.05, A_{2}$ $=0.71 \pm 0.06$. In the system shown, the PIPT is completed within $20 \mathrm{~min}$, and a photon entry into the system of no $(\mathrm{ph})=3.5 \times 10^{19}$ leads to a similar ratio of number of photons to dye molecules of about no(ph): no(dye) $=9 \times 10^{2}: 1$. The quasi-stationarity of the system is reached after $40 \mathrm{~min}$ of illumination, corresponding to $\mathrm{no}(\mathrm{ph})=1 \times 10^{19}$ absorbed photons into the system. In general, in the $30 \%$ system the PIPT is not as efficient as in the $35 \%$ system.

Figure 11 (bottom) summarizes the time behavior of the full width at half maximum of the diffuse scatter signal of the ME with respect to the reflection of the LC phase. For the first, a monoexponential decay law with $\tau_{\mathrm{FWHM}, \mathrm{ME}}$ $=10 \pm 5$ min could be fitted, for the last a time constant of $\tau_{\mathrm{FWHM}, \mathrm{ME}}=7 \pm 2 \mathrm{~min}$. Also these time dependencies indicate a fast PIPT of the system within the first $20 \mathrm{~min}$ of illumination time. The FWHM of the ME scatter signal decreases from about $\Delta q_{\mathrm{FWHM}}=3 \AA^{-1}$ to $\Delta q_{\mathrm{FWHM}}=2.5 \AA^{-1}$; the FWHM from the LC phase increases to $\Delta q_{\mathrm{FWHM}}$ $=0.004 \AA^{-1}$.

The refinement of the LC reflection according to the Porod equation [Eq. (4)] yields the time evolution of the number of lamella $\left[N_{\text {lamella }}\right.$ (Fig. 12, top) $]$ and periodicity of the system $\left[d_{\text {lamella }}\right.$ (Fig. 12, bottom) $]$ for the system $C_{8} E_{4}, \gamma$ $=35 \%$. Within the first $10 \mathrm{~min}\left(\tau_{N, \text { lamella }}=-7 \pm 0.1 \mathrm{~min}\right)$, the number of lamella in the PIPT formed LC phase increases from 0 to $N_{\text {lamella }}=16 \pm 2$. After that, the reflection peak broadens considerably to the phase segregation process and a refinement of $N_{\text {lamella }}$ is not meaningful anymore. Furthermore, within the LC phase the periodicity constant rises up to $d_{\text {lamella }}=76.8 \pm 0.5 \AA$ and stays at that value before the phase segregation takes place. The time dependence of $d_{\text {lamella }}$ was fitted to $\tau_{d, \text { lamella }}=5 \pm 0.1 \mathrm{~min}$.

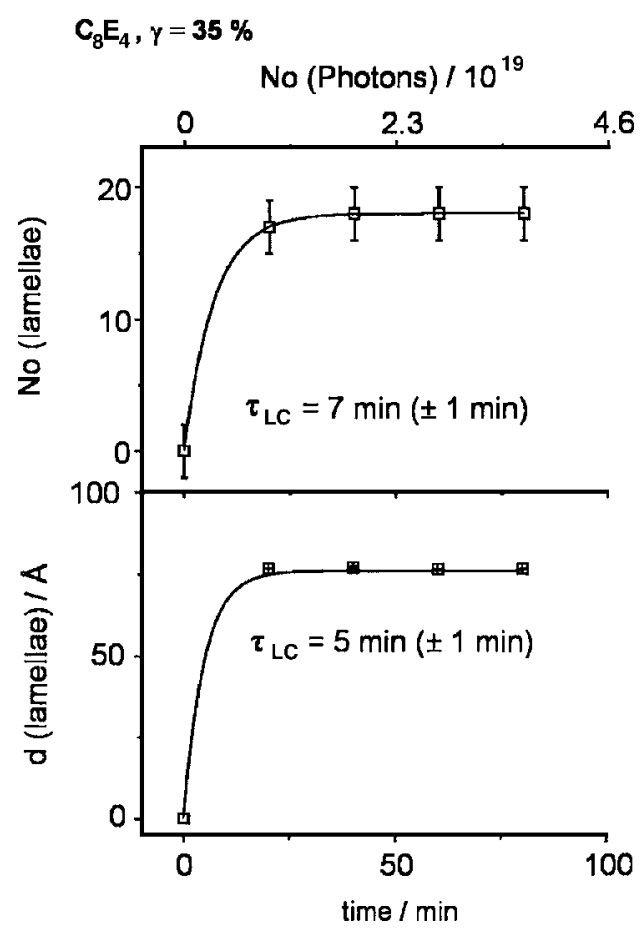

FIG. 12. System: $\mathrm{C}_{8} E_{4} /$ water/octane/cyclohexane, $\gamma=35 \%$. Phase segregation to two LC domains after 120 min of illumination indicated by a side reflection next to the main reflection of the initially formed LC phase.

In the $\gamma=30 \% \mathrm{C}_{8} E_{4}$ system (Fig. 13), within $\tau_{N \text {,lamella }}$ $=21 \pm 1 \mathrm{~min}$ the number of lamella increases from zero to $N_{\text {lamella }}=39 \pm 2$ (Fig. 13, top), indicating that the liquid crystal phase formed by PIPT is better ordered than the $\mathrm{C}_{8} E_{4}$,
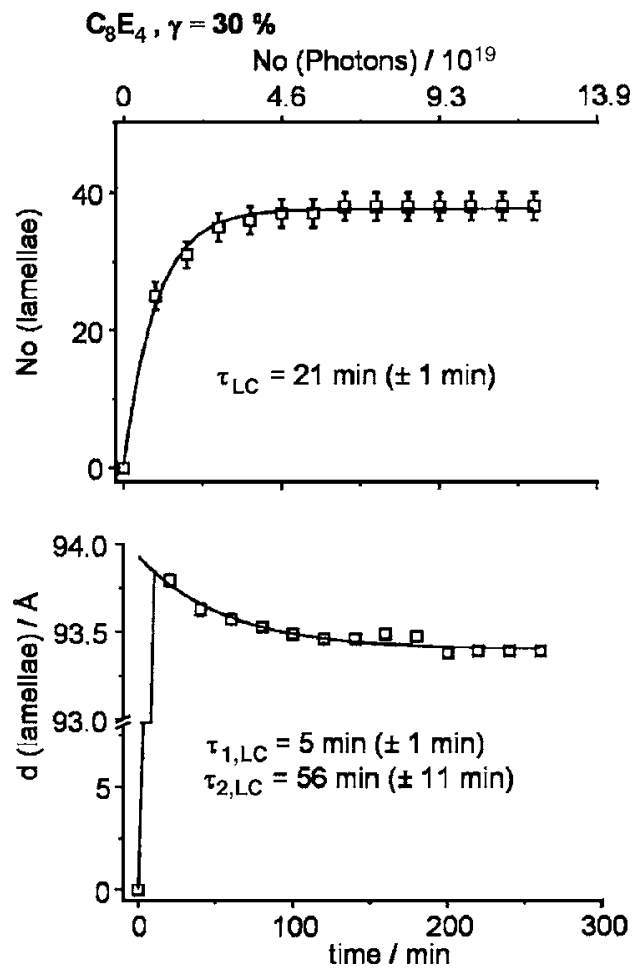

FIG. 13. System: $\mathrm{C}_{8} E_{4} /$ water/octane/cyclohexane, $\gamma=30 \%$. Top: During the formation of the liquid crystal phase, the number of lamella increases from 0 to 16 . Bottom: variation of $d$-spacing of the LC phase as a function of illumination time indicating volume contraction and therefore higher order of the LC phase at longer time scales. 
$\gamma=35 \%$ system under the same excitation conditions and therefore leading to bigger domain units within the LC phase. The reflections remain quite sharp during longer illumination time and the quality of the collected diffraction data is sufficient to study the system on longer time scales. After the first $20 \mathrm{~min}$ of illumination, the LC scattering peak rises up $\left(\tau_{1, d_{\text {lamella }}}=5 \pm 1 \mathrm{~min}, A_{1}=92.8 \pm 0.36\right)$ whose characteristic periodicity constant $d$ is $d_{\text {lamella }}=93.8 \pm 0.02 \AA$ (Fig. 13, bottom). For longer illumination time the lattice constant has been decreased to $d_{\text {lamella }}=93.25 \pm 0.02 \AA$. The decrease in the $d$ lattice constant indicates a volume contraction of the LC system and an additional ordering process of the LC system after the PIPT. The time constant of that volume contraction takes place on a long time scale of $\tau_{2, d_{\text {lamella }}}$ $=56 \pm 12 \mathrm{~min} \quad\left(A_{2}=0.53 \pm 0.06\right)$. A comparison to temperature-dependent SAXS measurements reveals that this volume contraction corresponds to a temperature decrease of the system by $\Delta T=-0.2 \mathrm{~K}$. This phenomenon will be discussed later.

The characterization of the structural response function of the LC phase is completed by investigating the thickness of the lamella $L$. The definition of $L$ is shown in Fig. 1. Typical values for $L$ are $L=48 \AA$ corresponding to two times the length chain of a $\mathrm{C}_{8} E_{4}$ surfactant molecule $(24 \AA)$. For the system $\mathrm{C}_{8} E_{4}$ in water/octane/cyclohexane with $\gamma=35 \%$, $L$ is nearly constant upon illumination time while the lattice constant $d$ decreases (Table I). The decrease in $d$ upon the formation of the LC phase due to PIPT can only be geometrically explained by a tighter ordering of the lamella layers as the liquid crystal phase is formed.

\section{Structural mechanism underlying the PIPT}

The photoillumination experiments carried out at the fluorescence spectrometer and the time-resolved photoSAXS apparatus suggest that the anti-Stokes excitation of the Rh101 dye in $\mathrm{C}_{8} E_{4}$ microemulsion systems results in a PIPT starting from local effects of energy entry into the system to the whole bulk reorganizing afterward. The PIPT is homogeneous and reversible since the ME phase recovers fully back after switching off the laser system. Near the thermal equilibrium, the investigated phase transition is purely controlled by the ratio of the number of incoming optical photons/absorbed photons and the number of photoexcitable dye molecules. The characteristic time constant of the PIPT is faster than $5 \mathrm{~min}$; the PIPT to the LC phase has finished after $20 \mathrm{~min}$.

For the photosensitization, the water soluble Rh101 dye was chosen with a quantum yield near unity in the ME phase. Exciting the dye with $632.8 \mathrm{~nm}$ in the fluorescence anti-Stokes regime leads to an excitation near the vibrational (0-0) transition of the vibronic $S_{1} \leftarrow S_{0}$ transition. The fluorescence is driven by energy transfer mechanisms based on the collision of the dye molecules first with its surrounding neighboring molecules. The energy entry to the dye molecules allows for intramolecular vibrational redistribution processes as some kind of up-conversion processes in the dye molecule so that the optically resonant $S_{1}$ state $(v=0)$ is populated from which fluorescence is possible. The energy entry into the dye's system leads to their fluorescence, the energy entry from the neighboring molecules to redistribution processes in the environment to compensate for the energy loss to the dye molecule. The redistribution results first in a restructuring of the microenvironment (as can be seen in the time dependence of the shift in fluorescence maxima), which leads to a complete switch of the phase to the LC phase. However, as one can see from the studies of the temperature dependence of the dye system in the ME and LC phases, the quenching of the fluorescence quantum yield to 0.6 suggests that the mechanism described above only holds for $60 \%$ of the Rh101 molecules excited. The remaining $40 \%$ of excited molecules perform ordinary internal conversion processes or form excimerlike structures, as has been suggested in the analysis of the time-resolved fluorescence measurements above. Indeed, an analysis of the photo-SAXS diffraction pattern of the well-ordered $\mathrm{C}_{8} E_{4}$ system with $\gamma$ $=30 \%$ shows that the characteristic periodicity constant $d$ of the LC phase shrinks from $d_{\text {lamella }}=93.8 \pm 0.02 \AA$ to $d_{\text {lamella }}$ $=93.25 \pm 0.02 \AA$ at a time scale of $\tau_{d \text {,lamella }}=56 \pm 12 \mathrm{~min}$. The whole mechanism coincides with a shearing of the lamella away from each other on the same time scale. On the other side, the intensity variation in the fluorescence spectra are described by a biexponential time law with a very fast time constant of $\tau_{1}=5 \mathrm{~min}$ and $\tau_{2}=120 \mathrm{~min}$. The restructuring of the PIPT formed phase on this long time scale leads in the case of the $\mathrm{ME} \rightarrow \mathrm{LC}$ PIPT to a higher order in the lamellar stacking supporting the formation excimers quenching the monomer fluorescence. By comparing the Bragg peak shift of the LC phase of the photo-SAXS experiments with static temperature-dependent SAXS measurements, the lattice constant decrease corresponds to a final temperature decrease of the system by about $\Delta T=-0.2 \mathrm{~K}$.

The characteristic time scales for the energy redistribution processes after optical excitation $(632.8 \mathrm{~nm})$ where energy is redistributed from the dye system to its local surrounding via collisions and vibrational relaxations are femtoto picosecond time scales. The rate limiting step is the lifetime of the Rh101 dye, which is about $6.2 \mathrm{~ns}$ for the antiStokes process. If the number of absorbed photons reaches an excess of $10^{4}$ compared to the number of dye molecules, the energy redistribution based on these very fast effects into the system is sufficient to induce a PIPT in the whole bulk. Again, the time constant of about $5 \mathrm{~min}$ for the PIPT is purely rate determined by the flux of the optical photons. The energy entry induces a vertical PIPT transition in the phase diagram of the ME-LC system followed by a restructuring of the LC phase to higher packing (120 min time scale) or induces degradation. It has furthermore been observed by the TR photo-SAXS technique that on a long time scale of more than 800 min of illumination time, a considerable degradation of the system takes place. During that degradation the long-range order of the liquid crystal phase got lost and the liquid crystal reflection disappears. For technical applications (like liquid crystal lasers), these degradation effects therefore have to be considered. 


\section{CONCLUSION}

The experiments and the sensitization method demonstrated open a novel route for photoswitching bulk matter and give a new possibility for the optical switching of electronic devices like liquid crystal systems. Time-resolved spectroscopic techniques and time-resolved photo-SAXS techniques reveal that the photoinduced structural changes lead to a structural phase transition of the bulk from the microemulsion phase to the liquid crystal phase. The PIPT of bulk microemulsion of $1 \mathrm{~mm}^{3}$ volume occurs within some minutes and is purely rate limited by the flux of the incoming optical photons and its ratio to the number of dye molecules. After the formation of the LC phase due to PIPT, the system undergoes a restructuring process to a higher ordered LC phase (in $120 \mathrm{~min}$ ). Long time illumination over one to two days leads to a degradation of the sample. Without photosensitization these systems are optically transparent and not photoswitchable. The PIPT from the microemulsion phase to the liquid crystal phase is reversible, and the microemulsion signal recovers after switching of the laser.

\section{ACKNOWLEDGMENTS}

This work was supported by DFG/SFB755 Nanoscale Photonic Imaging. S.T. is grateful to the DFG (TE347, 1-3), Aventis Foundation and Fonts of the Chemical Industry. W.Q. thanks the DAAD for financial support. The Advanced Study Group of the Max Planck Society is thanked for continuous support. J. Davaasambuu is thanked for the initial help in the work.

${ }^{1}$ P. Gütlich, A. Hauser, and H. Spiering, Angew. Chem., Int. Ed. Engl. 33, 2024 (1994).

${ }^{2}$ K. Nasu, Relaxation of Excited States and Photo-Induced Structural Phase Transitions (Springer-Verlag, Berlin, 1997).

${ }^{3}$ S. Koshihara, Y. Takahashi, H. Sakai, Y. Tokura, and T. Luty, J. Phys. Chem. B 103, 2592 (1999).

${ }^{4}$ E. Collet, M. H. Lemee-Cailleau, M. Buron-Le Cointe, H. Cailleau, M. Wulff, T. Luty, S. Y. Koshihara, M. Meyer, L. Toupet, P. Rabiller, and S Techert, Science 300, 612 (2003).

${ }^{5}$ M. Kahlweit, Ber. Bunsenges. Phys. Chem. 98, 490 (1994).

${ }^{6}$ X. L. Zhou, L. T. Lee, S. H. Chen, and R. Strey, Phys. Rev. A 46, 6479 (1992).

${ }^{7}$ S. Chandrasekhar and N. V. Madhusudana, Annu. Rev. Mater. Sci. 10, 133 (1980).

${ }^{8}$ D. Roux, C. Coulon, and M. E. Cates, J. Phys. Chem. 96, 4187 (1992).
${ }^{9}$ E. Z. Radlinska, G. Krzywicki, F. Lafuma, D. Langevin, W. Urbach, C. E. Williams, and R. Ober, Phys. Rev. Lett. 74, 4237 (1995).

${ }^{10}$ M. Monkebusch, O. Holderer, H. Frielinghaus, D. Byelov, J. Allgaier, and D. J. Richter, J. Phys.: Condens. Matter 17, 2903 (2005).

${ }^{11}$ L. Corvazier, L. Messe, C. Salou, R. Young, J. Patrick, A. Fairclough, and A. J. Ryan, J. Mater. Chem. 11, 2864 (2001).

${ }^{12}$ D. Byelov, H. Frielinghaus, O. Holderer, J. Allgaier, and D. J. Richter, Langmuir 20, 10433 (2004).

${ }^{13}$ F. Reinitzer, Monatsch. Chem. 9, 421 (1888).

${ }^{14}$ O. Lehmann, Flüssig-Kristalle sowie Plastizität von Kristallen im Allgemeinen, Molekulare Umwandlungen und Aggregatzustandsänderungen (W. Engelmannsverlag, Leipzig, 1904).

${ }^{15}$ C. Zander and H. Drexhage, Advances in Photochemistry, C. D. Neckers, D. H. Volman, and G. von Bünau (Eds.) (Wiley, New York, 1995).

${ }^{16}$ B. Stevens, N. Connelly, and P. Suppan, Spectrochim. Acta 22, 2121 (1966).

${ }^{17}$ J. L. Clark and G. Rumbles, Phys. Rev. Lett. 76, 2037 (1996).

${ }^{18}$ C. E. Mungan, M. I. Buchwald, B. C. Edwards, R. I. Epstein, and T. R. Gosnell, Phys. Rev. Lett. 78, 1030 (1997).

${ }^{19}$ S. Techert, S. Schmatz, A. Wiessner, and H. Staerk, J. Phys. Chem. A 104, 5700 (2000).

${ }^{20}$ W. Quevedo, M. Petri, and S. Techert, Z. Kristallogr. 223, 43 (2008).

${ }^{21} \mathrm{G}$. Busse, Th. Tschentscher, A. Plech, M. Wulff, B. Frederichs, and S. Techert, Faraday Discuss. 122, 105 (2003).

${ }^{22}$ S. Techert, J. Appl. Crystallogr. 37, 445 (2004).

${ }^{23}$ S. Techert and S. Schmatz, Z. Phys. Chem. 216, 575 (2002).

${ }^{24}$ G. Busse, B. Frederichs, N. Petrov, and S. Techert, Phys. Chem. Chem. Phys. 6, 3309 (2004).

${ }^{25}$ A. S. F. Ramos and S. Techert, Biophys. J. 89, 1990 (2005).

${ }^{26}$ A. M. Lindenberg, J. Larsson, K. Sokolowski-Tinten, K. J. Gaffney, C. Blome, O. Synnergren, J. Sheppard, C. Caleman, A. G. MacPhee, D. Weinstein, D. P. Lowney, T. K. Allison, T. Matthews, R. W. Falcone, A. L. Cavalieri, D. M. Fritz, S. H. Lee, P. H. Bucksbaum, D. A. Reis, J. Rudati, P. H. Fuoss, C. C. Kao, D. P. Siddons, R. Pahl, J. Als-Nielsen, S. Duesterer, R. Ischebeck, H. Schlarb, H. Schulte-Schrepping, Th. Tschentscher, J. Schneider, D. von der Linde, O. Hignette, F. Sette, H. N. Chapman, R. W. Lee, T. N. Hansen, S. Techert, J. S. Wark, M. Bergh, G. Huldt, D. van der Spoel, N. Timneanu, J. Hajdu, R. A. Akre, E. Bong, P. Krejcik, J. Arthur, S. Brennan, K. Luening, and J. B. Hastings, Science 308, 392 (2005).

${ }^{27}$ C. Blome, Th. Tschentscher, J. Davaasambuu, P. Durand, and S. Techert, J. Synchrotron Radiat. 12, 812 (2005).

${ }^{28}$ P. Coppens, I. Novozhilova, and A. Kovalevsky, Chem. Rev. (Washington, D.C.) $\mathbf{1 0 2}, 861$ (2002)

${ }^{29}$ J. Cole, Chem. Soc. Rev. 33, 501 (2004).

${ }^{30}$ S. Techert and K. A. Zachariasse, J. Am. Chem. Soc. 126, 5593 (2004).

${ }^{31}$ L. X. Chen, W. J. H. Jäger, G. Jennings, D. J. Gosztola, A. Munkholm, and J. P. Hessler, Science 292, 262 (2001).

${ }^{32}$ A. S. F. Ramos and S. Techert, Phys. Chem. Chem. Phys. 5, 5176 (2003).

${ }^{33}$ O. Glatter and O. Kratky, Small Angle X-Ray Scattering (Academic, New York, 1982).

${ }^{34}$ M. Teubner and R. Strey, J. Chem. Phys. 87, 3195 (1987). 